\section{Cost effectiveness of the type II Boston keratoprosthesis}

\begin{abstract}
Purpose Despite demonstrated cost effectiveness, not all corneal disorders are amenable to type I Boston keratoprosthesis (KPro) implantation. This includes patients with autoimmune diseases, such as Stevens-Johnson syndrome/toxic epidermal necrolysis. Type II KPro is implanted through the eyelids in severe dry eye and cicatricial diseases, and its cost effectiveness was sought. Patients and methods In a retrospective chart review, 29 patients who underwent type II KPro surgery at the Massachusetts Eye and Ear Infirmary between the years 2000 and 2009 were identified. A total of 11 patients had 5-year follow-up data. Average cost effectiveness was determined by cost-utility analysis, comparing type II KPro surgery with no further intervention.

Results Using the current parameters, the cost utility of KPro from third-party insurer

(Medicare) perspective was 63196 \$/qualityadjusted life year.

Conclusion Efforts to refer those less likely to benefit from traditional corneal

transplantation or type I KPro, for type II KPro surgery, may decrease both patient and societal costs.

Eye (2011) 25, 342-349; doi:10.1038/eye.2010.197; published online 24 December 2010
\end{abstract}

Department of

Ophthalmology,

Massachusetts Eye and Ear Infirmary, Harvard Medical School, Boston, MA, USA

Correspondence: JD Ament, Department of

Ophthalmology,

Massachusetts Eye and Ear Infirmary, Harvard Medical School, 243 Charles Street, Boston, MA, 02114-4724, USA

Tel: + 16177214673 ;

Fax: +1 6175734300

E-mail: jaredament@

post.harvard.edu

Received: 4 May 2010

Accepted in revised form:

8 November 2010

Published online:

24 December 2010

This work was presented as a poster at the World

Cornea Congress Meeting in Boston, April 2010.
Keywords: decision analysis; cost utility; cost effectiveness; keratoprosthesis; autoimmune diseases

\section{Introduction}

Type I Boston keratoprosthesis (KPro) has recently been shown to be a highly cost-effective medical intervention at 16140 \$/qualityadjusted life years (QALYs). ${ }^{1}$ However, not all corneal diseases are amenable to or appropriate for type I implantation. Patients with autoimmune diseases, such as Stevens-Johnson
JD Ament, TP Stryjewski, S Pujari, S Siddique, GN Papaliodis, J Chodosh and CH Dohlman

syndrome/toxic epidermal necrolysis and mucous membrane pemphigoid have severe ocular surface diseases where destruction of the corneal epithelial stem cells located at the corneal limbus results in conjunctival invasion, corneal neovascularization, chronic inflammation, and stromal scarring. ${ }^{2}$ In end-stage cases, the ocular surface becomes completely dry and the fornices become obliterated. Corneal transplantation and type I KPro, in such cases, are almost inevitably associated with a poor prognosis., ${ }^{3,4}$ Patients' quality of life is appreciably affected, often experiencing considerable, if not complete, loss of vision and numerous comorbidities. Because of the destructive nature of these conditions, there have been few reports on the use of KPro surgery for the treatment of autoimmune diseases. ${ }^{5-8}$ In contrast, several manuscripts have described the modified osteoodontokeratoprosthesis as efficacious in this patient population. ${ }^{9-11}$ For many, this represents the gold standard for severe ocular surface disease. Nevertheless, the morbidity and postoperative complications associated with this lengthy, two-stage procedure, ${ }^{11}$ and the possibility for a more simplistic and pragmatic KPro cannot be ignored, especially as recent modifications to the type II design and postoperative management of patients have led to improved clinical outcomes. ${ }^{12,13}$

To determine cost effectiveness by way of cost-utility analysis (CUA), the perceived value of an intervention or health state is taken into account. Utilities on a scale from 0 to 1 are generated, most commonly by the time trade-off approach, ${ }^{14,15}$ and these are used to determine overall benefit. Benefit is measured in terms of QALYs.

To our knowledge, no CUA has been performed on the use of the type II Boston KPro. It is the intention of this study to objectively assess the (1) comparative effectiveness (gain in QALYs) and (2) average cost effectiveness (compared with no further treatment or current 
visual state) of the type II Boston KPro procedure for the treatment of severe corneal disease and blindness.

\section{Patients and methods}

\section{Boston KPro type II}

Type II Boston KPro was developed at the Massachusetts Eye and Ear Infirmary. ${ }^{16}$ As seen in Figures 1 and 2, it is a collar button shaped polymethylmethacrylate device consisting of two curved plates that is implanted through the eyelids in severe dry eye and cicatricial diseases. Type II Boston KPro is performed far less than type I, and is reserved for near-hopeless cases with severe destruction of the ocular surface.

\section{Patients}

In a retrospective cohort study chart review, 29 patients who underwent type II KPro surgery at the Massachusetts Eye and Ear Infirmary between the years 2000 and 2009 were identified. Patients had to have a minimum of 5 years of visual acuity follow-up data recorded. Patients receiving

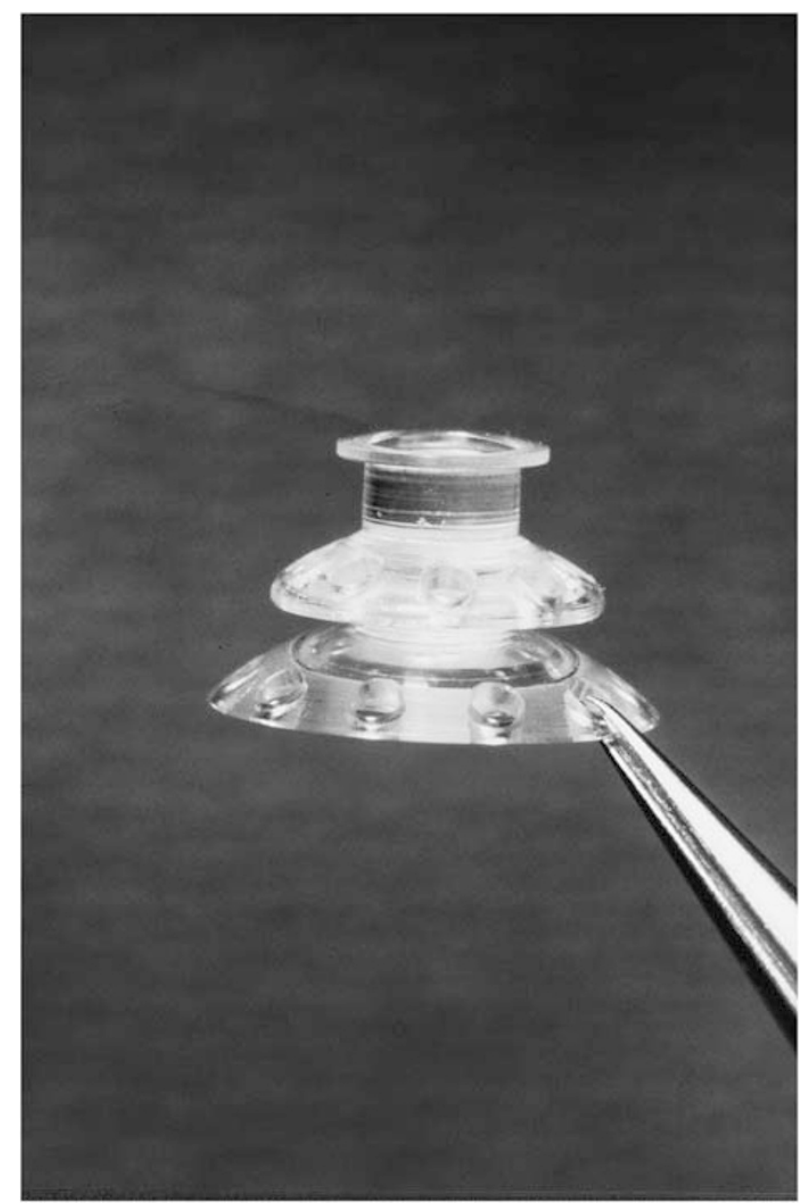

Figure 1 The type II Boston keratoprosthesis. The front plate of the keratoprosthesis is shown assembled ex vivo. type II KPro were in the worst prognostic groups, such as autoimmune diseases and chemical burns. A total of 11 patients had a minimum of 5 years of follow-up and were included in the study.

Patient characteristics with underlying diagnoses before KPro surgery are given in Table 1. A complete ophthalmic exam was performed before KPro surgery. Median preoperative best-corrected visual acuity (BCVA) in the treated eye was $\log$ MAR $2.3 \pm 0.7$ (Snellen equivalent HM). Visual acuity values were normally distributed per Shapiro-Wilk testing. We certify that all the applicable institutional and governmental regulations concerning the ethical use of human volunteers were followed during this research.

\section{Decision analysis}

Average cost effectiveness was determined, comparing type II KPro surgery with no further intervention (the current visual state). Complications and additional procedures were incorporated into an expected-value

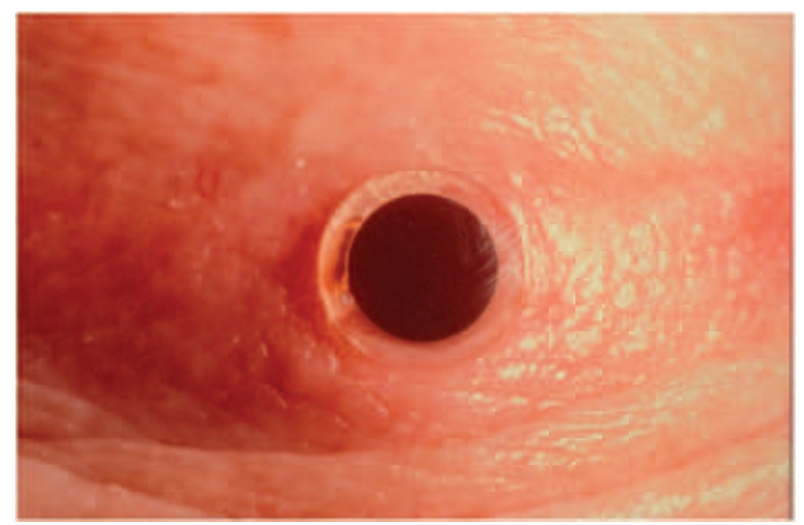

Figure 2 The clinical appearance of the type II Boston keratoprosthesis. The front plate of the keratoprosthesis is shown implanted through the eyelid of a patient with a history of Steven-Johnson's Syndrome.

Table 1 Characteristics of type II Boston KPro patients included in this study

\begin{tabular}{lcc}
\hline Characteristic & Amount & Qualifier \\
\hline $\begin{array}{l}\text { Patients } \\
\text { Gender }\end{array}$ & $\begin{array}{c}n=11 \\
\text { Male }=6 ; \\
\text { female }=5 \\
\text { Mean }=66 \text { years }\end{array}$ & Range $=37-86$ years \\
Age & $n=6$ & $54.5 \%$ \\
$\begin{array}{l}\text { Diagnostic group } \\
\begin{array}{l}\text { Stevens-Johnson } \\
\text { syndrome }\end{array}\end{array}$ & $n=4$ & $36.4 \%$ \\
$\begin{array}{l}\text { Ocular cicatricial } \\
\text { pemphigoid } \\
\text { Chemical burns }\end{array}$ & $n=1$ & $9.0 \%$ \\
\hline
\end{tabular}

Abbreviation: Kpro, keratoprosthesis. 
Table 2 Postoperative complications and procedures over 5 years for type II Boston KPro recipients in this study

\begin{tabular}{lccc}
\hline Complication/procedure & No. of patients & No. of treatments & $\begin{array}{c}\text { Percentage of total cohort } \\
\text { (based on treatment) }\end{array}$ \\
\hline Retroprosthetic membranes & & 7 YAGs & $63.6 \%$ \\
& 5 & 1 membranectomy & $9.1 \%$ \\
Corneal leak & 6 & 7 revisions & $63.6 \%$ \\
Inflammation & 6 & 7 kenalog injections & $63.6 \%$ \\
High IOP & 7 & 7 Ahmed valves & $63.6 \%$ \\
Skin overgrowth & 3 & 4 skin revisions & $36.4 \%$ \\
Decrease in visual acuity & 2 & 2 revisions & $18.2 \%$ \\
Necrosis & 1 & 2 revisions & $18.2 \%$ \\
Systemic immunosuppressive therapy & 3 & 2 systemic steroids & $18.2 \%$ \\
& & 1 alkylating agent & $9.1 \%$ \\
Retinal detachment & 3 & 1 antimetabolites & $9.1 \%$ \\
Endophthalmitis (infectious) & 1 & 1 PPV + silicone oil & $9.1 \%$ \\
& & 1 IV vanc. & $9.1 \%$ \\
Cellulitis & & 1 intraocular vanc. & $9.1 \%$ \\
Tarsorrhaphy & 1 & 1 amphotericin B & $9.1 \%$ \\
KPro extrusion & 1 & 1 medical regimen & $9.1 \%$ \\
\hline
\end{tabular}

Abbreviations: IOP, intraocular pressure; Kpro, keratoprosthesis; PPV, pars plana vitrectomy; vanc, vancomycin; YAG, yttrium aluminium garnet.

decision tree. A list of the most common postoperative complications and treatments are given in Table 2.

To make cost-utility calculations, several model assumptions were made.

In our sample, 5-year anatomical retention was $72.7 \%$. (1) The time frame chosen for the CUA was 5 years because of presumed quiescence of disease at this postoperative time; (2) a yearly KPro implant survival probability of $93.5 \%$ was conservatively interpolated from the data; (3) BCVA preoperatively and at 5 years postoperatively was utilized to calculate incremental utilities; (4) the average incremental utility of the 5-year cohort represented that of the entire sample; (5) the mean patient age was 66, and we postulated that this was representative of this population.

\section{Utility assessment}

The time trade-off method was used for patient-based utility assessment in the CUA. The mean $( \pm$ SD) preoperative utility value was 0.391 ( $\pm 0.1 .36)$, increasing to $0.568( \pm 0.224)$ at 5 years postoperatively. Decreases in vision were accounted for by incorporating negative utilities into the mean incremental utility calculation. The mean incremental utility in our study population at 5 years was 0.177 . The total QALY gain (comparative effectiveness) was also determined by multiplying the years of utility gain by years of benefit duration and comparing it with the preoperative utility (quality of life) state.

\section{Identification of costs}

The costs for the KPro type II device, surgical procedure, hospitalization, and follow-up management were obtained from the Massachusetts Eye and Ear Infirmary CPT diagnosis and procedure codes. The CUA was conducted based on the Medicare reimbursement rates in 2009. Ophthalmologic visits were conducted every 3 months for the first year, followed by every 6 months over the second year, and then annually. Maintenance costs included medications and patient travel. An overview of all costs is given in Table 3.

The expected-value decision tree is illustrated in Figure 3. Future costs, such as follow-up visits and possible complications requiring procedures, are weighted based on normal clinical practice and rates observed in our sample (refer to Table 2). Total costs were calculated by the summation of initial costs with appropriately discounted future costs. Discounted future costs included inevitable costs and the average of weighted probable/possible future costs.

\section{Discounting}

There is a consensus that both utilities and costs should be discounted in health care economics analyses. We decided that the commonly used $3 \%$ discount rate was appropriate. ${ }^{17}$

\section{Calculation of QALYs}

The QALYs for the 5-year period was calculated using the following formula: ${ }^{18,19}$

$$
\sum_{x=1}^{5} \frac{t^{x} u}{\left(1+d_{q}\right)^{x}}
$$

where $t$ is the yearly transplant survival rate (0.935), $u$ is the average incremental utility $(0.177)$, and $d_{q}$ is the discounting rate for QALYs (3\%). 
Table 32010 Medicare reimbursement schedule associated with Boston Kpro type II

\begin{tabular}{|c|c|c|}
\hline Item or Service & CPT code & Cost (USD) \\
\hline Evaluation & 92004 & 100 \\
\hline KPro surgery (surgeon fee) & 65770 & 1401 \\
\hline Facility fee + cornea & - & $10431^{a}$ \\
\hline ECCE without $\mathrm{IOL}^{\mathrm{a}}$ & 66984 & 896 \\
\hline Glaucoma shunt $^{\mathrm{a}}$ & 66180 & 1577 \\
\hline Tarsorrhaphy $^{\mathrm{a}}$ & 67880 & 618 \\
\hline Follow-up visit & 99211 or 99212 & 200 per year \\
\hline $\begin{array}{l}\text { Antibiotic medications } \\
\left(\text { vanc } / \mathrm{pf} / \text { moxi }^{\mathrm{b}}\right)\end{array}$ & - & $600 /$ year \\
\hline $\begin{array}{l}\text { Immunosuppressive } \\
\text { medications (mycophenolate } \\
\text { mofetil/infliximab) }\end{array}$ & - & 16400/year \\
\hline Patient travel & - & 100/year \\
\hline \multicolumn{3}{|l|}{ Procedures related to complications } \\
\hline YAG & 67031 & 333 \\
\hline Membranectomy & 65865 & 1223 \\
\hline Kenalog injection & 67500 & 147 \\
\hline Shunt revision ${ }^{\mathrm{a}}$ & 66185 & 1837 \\
\hline Silicone oil ${ }^{\mathrm{a}}$ & 67042 & 1417 \\
\hline Vitrectomy $^{\mathrm{a}}$ & 67036 & 1408 \\
\hline Iris repair & 66680 & 919 \\
\hline Strabismus surgery & 67331 & 1967 \\
\hline Endophthalmitis ${ }^{c}$ & $65810 / 66250 / 66020$ & 3000 \\
\hline
\end{tabular}

Abbreviations: CPT, current procedural terminology; IOL, intra-ocular lens; Kpro, keratoprosthesis; moxi, moxifloxacin; pf, prednisolone acetate 1\%; USD, United States Dollars; vanc, vancomycin.

aSome procedures (ie, vitrectomy, silicone oil injection, cost of KPro, and anesthesia) are bundled into KPro or facility fee, and additional procedures at the time of surgery are reimbursed at $50 \%$.

${ }^{\mathrm{b}}$ Vancomycin, prednisolone acetate $1 \%$, moxifloxacin - the standard postoperative regimen

'Infectious endophthalmitis management in type II KPro patients usually requires the operating room, tarsorrhaphy revision, paracentesis of the eye for culture, injection of vancomycin $1 \mathrm{mg}$, ceftazidime $2.25 \mathrm{mg}$, and amphotericin B $5 \mathrm{mcg}$.

\section{Calculation of cost}

The equation for the total discounted cost associated with KPro surgery is:

$$
\begin{aligned}
& \$ 11932+\sum_{x=1}^{5} \frac{\$ 600}{\left(1+d_{c}\right)^{x}}+\sum_{x=1}^{5} \frac{\$ 200}{\left(1+d_{c}\right)^{x}} \\
& +\sum_{x=1}^{5} \frac{\$ 2991}{\left(1+d_{c}\right)^{x}}+\frac{\$ 14979}{\left(1+d_{c}\right)^{5}},
\end{aligned}
$$

An initial cost that was incurred at or immediately before or following the time of surgery was not discounted. Costs paid for over the initial year alone were discounted accordingly as were costs paid for throughout the entire time period. In the equation, $x$ represents the year of follow-up and $d_{c}$ is the discounted rate for costs $(3 \%)$.

\section{Sensitivity analysis}

The model was assessed using a univariate sensitivity analysis (Table 4.). The relevant parameters included utility value, retention rate, discounting rate for QALYs, and discounting rate for costs. Each parameter was varied at fixed intervals individually.

\section{Results}

Median preoperative BCVA in the treated eye was $\log$ MAR $2.3 \pm 0.7$ (Snellen equivalent HM). At 5 years postoperatively, the median BCVA increased to logMAR $1.30 \pm 1.17$ (Snellen equivalent of $20 / 400$ ). A total discounted incremental QALY gain of 0.668 was obtained for type II KPro. This correlates with a conferred QALY gain (or improvement in quality of life) of $8.7 \%$ for the average patient. The total discounted cost associated with this utility equaled $\$ 42215$. Using the current parameters, the cost utility of KPro from third-party insurer (Medicare) perspective was 63196 \$/QALY. The univariate sensitivity analysis resulted in a range of incremental cost-effectiveness ratios from 52078 to 83871 \$/QALY.

\section{Discussion}

As noted in the paper by Ament et al., describing the cost effectiveness of type I Boston KPro, the commonly cited guideline considers interventions costing below 20000 \$/QALY as highly cost effective and interventions costing more than 100000 \$/QALY as not cost effective. ${ }^{20}$ The UK National Institute for Health and Clinical Excellence (NICE) uses 60000 \$/QALY to define cost-effective treatments. ${ }^{21}$ It is nevertheless recognized that these benchmarks and the unit \$/QALY, as a measure of value in medicine, are inherently limited. Indeed, insurance companies and national health boards often rebuff reimbursement below these guidelines, fund beyond them, or develop novel pricing arrangements to expand access of otherwise less cost effective interventions. ${ }^{22}$ Various cost-effective values (\$/QALY) for several medical interventions are illustrated in Table 5.

In this analysis, only patients with 5 years of follow-up data were included. Although the 5-year sample was small, it was determined that 2- to 3-year follow-up was insufficient for this population. Based on anecdotal evidence, severe complications remain a concern well after the 2-year postoperative period in autoimmune patients undergoing type II KPro surgery. This is unlike type I KPro, in which visual gains can ostensibly be maintained almost indefinitely. Despite this, it is important to note that those patients considered eligible 


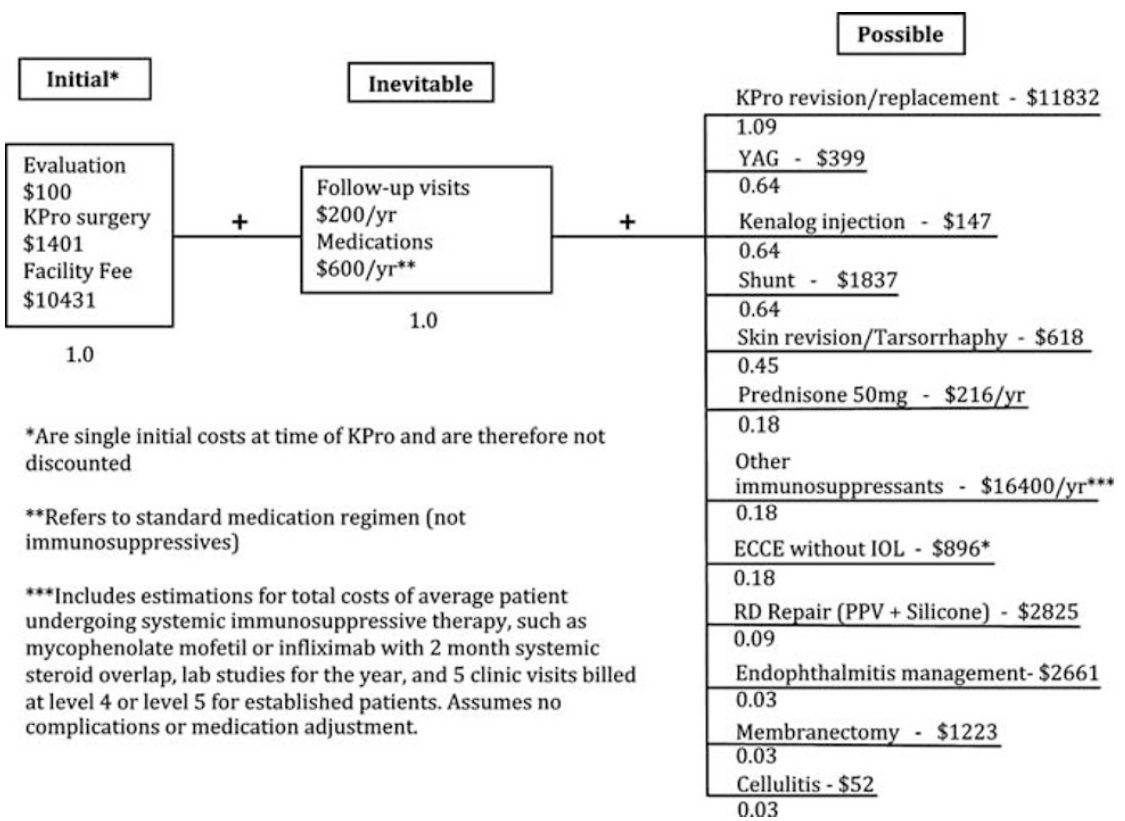

Figure 3 Expected value decision tree for the type II Boston keratoprosthesis - preoperative, perioperative, and postoperative management. Decision tree is broken down into initial, inevitable, probable, and possible costs. Costs are reported on the basis of the 2010 allowable reimbursement from Medicare. Probability of occurrence is displayed below the decision/cost item. A single asterisk indicates costs that require appropriate discounting. Discounting can occur over some or all of the years being assessed in this study, depending on need and utilization of the service. Furthermore, in some instances, the cost changes after the initial year of use.

Table 4 Univariate sensitivity analysis of the cost-utility analysis for type II Boston Kpro

\begin{tabular}{lcccc}
\hline $\begin{array}{l}\text { Variable adjustment } \\
\text { cost yield }\end{array}$ & $\begin{array}{c}\text { Yearly Boston Kpro } \\
\text { survival probability }\end{array}$ & $\begin{array}{c}\text { Incremental } \\
\text { utility value }\end{array}$ & Discount rate for QALY & Discount rate for costs \\
\hline High $^{\mathrm{a}}$ & 0.842 & 0.160 & 0.05 & 0.01 \\
Current & 0.935 & 0.177 & 0.03 & 0.03 \\
Low & 1.000 & 0.194 & 0.01 & 0.05 \\
Range (\$/QALY) & $52078-83871$ & $57587-69824$ & $59714-66595$ & $60001-66741$ \\
\hline
\end{tabular}

Abbreviations: Kpro, keratoprosthesis; QALY, quality-adjusted life year.

${ }^{a}$ Variables are changed by $10 \%$ from the current values.

for type II KPro implantation typically experience severe, debilitating sequellae of their underlying disease process, and may perceive a limited and transient 2-year improvement, irrespective of the complication rates and risks, as significant, thereby possessing inherent utility. Indeed, 16 patients had complete 2 years of follow-up and, on average, improved from $\mathrm{HM}$ vision to $20 / 70$. The average incremental utility increase for this cohort was exceedingly high at 0.278 . This, when compared with a 0.177 average incremental utility increase in our 5 -year cohort, represents a 57\% increase in average utility change. Reassessing 2-year costs and conducting the appropriate cost-utility calculations yield a cost-effective value of 31719 \$/QALY for this 2-year sample. These markedly disparate values illustrate that a dramatic change occurs in type II KPro population after the 2-year postoperative period. Although physiologic and pathologic processes need to be further elucidated, it is noteworthy that the cost-effective curve, as it relates to visual acuity, appears to be non-linear. Worsening vision and the associated substantial decrease in incremental utility disproportionately affect the cost-effective calculation as compared with only moderate increases in costs. Furthermore, vision changes from HM to 20/400, for example, are associated with a greater utility than, say, improvement from 20/200 to 20/20.

Despite the limited sample, the original 5-year study cohort demonstrated remarkable improvement in 
Table 5 Cost utility of various medical interventions in the US, adjusted to 2010 US dollars ${ }^{23}$

\begin{tabular}{|c|c|}
\hline Intervention (ophthalmology) & $\begin{array}{l}\text { Cost in } \\
\$ / Q A L Y\end{array}$ \\
\hline Initial cataract surgery $^{24}$ & 2023 \\
\hline Second eye cataract surgery ${ }^{25}$ & 2727 \\
\hline Penetrating keratoplasty ${ }^{19}$ & 12194 \\
\hline Boston Kpro type $\mathrm{I}^{1}$ & 16140 \\
\hline Boston Kpro type II (submitted paper) & 63196 \\
\hline \multicolumn{2}{|c|}{$\begin{array}{l}\text { Photodynamic therapy for subfoveal choroidal neovascularization with } \\
\text { ARMD }{ }^{26}\end{array}$} \\
\hline $20 / 40$ initial vision & 104158 \\
\hline 20/200 initial vision & 208966 \\
\hline \multicolumn{2}{|l|}{ Intervention (other) } \\
\hline TKR (high-risk patients) ${ }^{27}$ & 28381 \\
\hline $\begin{array}{l}\text { Antibiotic impregnated cement } \\
\text { compared with normal cement during hip } \\
\text { arthroplasty }^{28}\end{array}$ & 37595 \\
\hline $\begin{array}{l}\text { Coronary bypass surgery for occluded LAD } \\
\text { artery }^{29}\end{array}$ & 44113 \\
\hline $\begin{array}{l}\text { Chemoprophylaxis after occupational } \\
\text { exposure to HIV }{ }^{30}\end{array}$ & 49036 \\
\hline Primary pediatric heart transplant ${ }^{31}$ & 52417 \\
\hline Renal transplantation (in Greece) (32 $^{32}$ & 64966 \\
\hline $\begin{array}{l}\text { Incremental cost-utility ratio for TKR vs } \\
\text { unicompartmental knee arthroplasty }{ }^{33}\end{array}$ & 65979 \\
\hline Total hip arthroplasty (best/worst case) ${ }^{34}$ & $6353 / 110484$ \\
\hline $\begin{array}{l}\text { Magnetic resonance imaging for equivocal } \\
\text { neurologic symptoms }\end{array}$ & 134742 \\
\hline $\begin{array}{l}\text { Prophylactic hip fixation to prevent future } \\
\text { contralateral hip fracture }\end{array}$ & 142795 \\
\hline $\begin{array}{l}1 \text { day of chemoprophylaxis before receiving } \\
\text { dental work for patients with prosthetic } \\
\text { joints } \text { j }^{37}\end{array}$ & 696692 \\
\hline
\end{tabular}

Abbreviations: ARMD, age related macular degeneration; Kpro, keratoprosthesis; LAD, left anterior descending; QALY, quality-adjusted life year; TKR, total knee replacement

median visual acuity within 5 years from HM to 20/400 for a moderately cost-effective value of 63196 \$/QALY. Though optimistic for suggesting possible expansion of type II KPro for the management of patients with severe autoimmune ophthalmic conditions, the complexities of postoperative management should not be underestimated. Consequently, the limitations of this analysis require proper evaluation. Patients undergoing type II KPro surgery must commit to a twice-daily regimen of antibiotic eye drops for life, sometimes take toxic and expensive systemic immunosuppressive drugs, be willing to accept the cosmetic burden of the device, and be prepared for life-long follow-up with an experienced type II KPro surgeon. These requirements may be burdensome for some patients and therefore represent disutilities of the device not accounted for in this retrospective analysis. It is possible that the 0.668 QALYs that we observed would decrease in light of these disutilities and that the ultimate cost effectiveness of the device would be diminished. The lack of a validated testing mechanism to assess patient satisfaction and preferences postoperatively limits this and other cost-effective analyses.

Additional limitations of this analysis were its restriction to one location and the lack of available costutility literature to directly compare type II KPro with the modified OOKP - the latter being, arguably, the accepted standard of care outside the USA for this patient population. Nonetheless, the authors felt that astype II KPro is performed so infrequently and without adequate standardization outside of MEEI that including external type II KPro data into this analysis would have injected incalculable variation. With respect to the OOKP, only one study noted the cost of rehabilitating end-stage ocular surface disease with OOKP surgery (13661 pounds or 21786 USD). ${ }^{38}$ A case report by Geerling et al. ${ }^{9}$ did demonstrate an overall cost savings with the OOKP of $\sim 7400$ USD over 2 years. However, both of these only examined costs and savings of the OOKP, and did not include formal cost-utility analyses. Review of the literature suggests that no such analysis exists, making appropriate comparisons impossible at this time. Given the lack of comparative data and a large multicentered sample, external validity is limited, and it is difficult to determine whether the cost effectiveness of type II KPro will decrease or increase, as it becomes more utilized in the clinical setting.

Excluded from this cost analysis is a consideration of the costs incurred before type II KPro implantation. Of the 11 patients included in this analysis, 4 underwent previous penetrating keratoplasty, 3 underwent type I KPro implantation once, and 1 underwent type I KPro implantation twice, all of which failed. Additionally, seven patients had glaucoma valves in place at the time of surgery. In contrast, expensive systemic immunosuppressive therapies were assumed to be continued for the entire 5 years and may represent an overestimation of costs. Furthermore, a significant source of additional cost for type II KPro lies in the high probability of revision and/or replacement. This could be greatly mitigated by improving device design and biocompatibility, and exemplifies the need to revisit this analysis in the future. Taking the risks, complications, and morbidity associated with type II KPro into account, efforts to refer patients who are less likely to benefit from traditional corneal transplantation or type I KPro may be helpful in decreasing both the ultimate personal and societal cost of surgical intervention in these patients, as evidenced by the multiple, failed procedures that occur in this population. 


\section{Summary}

\section{What was known before}

- It was unclear whether type II Boston Kpro was a costeffective medical intervention. Type I had recently been shown to be highly cost effective; however, the patient population requiring type II is drastically different. These patients have severe autoimmune corneal disease and are exceedingly debilitated.

What this study adds

- This study does two things: (1) it confirms that type II Boston Kpro is cost effective (based on the $<100000$ \$/QALY convention and similar modalities); and (2) provides a cost-utility model from which adaptations, improvements, and refinements can be computed. It provides a foundation for which clinicians and insurers can speak about utility, value, and cost.

\section{Conflict of interest}

Dr Dohlman receives no personal profits from the sale of the Boston Keratoprosthesis. Proceeds go to the Massachusetts Eye and Ear Infirmary, and are used to support research and development. The authors are/were employees of the Massachusetts Eye and Ear Infirmary, and receive no financial benefit from the publication of this data. Dr Ament is a Clinical Research Fellow who is paid directly from Dr Dohlman's research and development fund.

\section{Acknowledgements}

We thank Kathy Colby, MD, PhD-Massachusetts Eye and Ear Infirmary and Ann Burke-Cost and Budget Manager. Both contributed to cost data.

\section{Author contributions}

Design of the study (JA, TS, SP, SS, JC, and CD); conduct of the study (JA, TS, SP, SS, JC, and CD); analysis and interpretation (JA, GP, JC, and TS); writing the article (JA and TS); critical revision (JA, TS, SP, SS, GP, JC, and $\mathrm{CD}$ ); final approval (JA, TS, SP, SS, GP, JC, and CD); data collection (JA, TS, SP, SS, and GP); provision of materials (SP, SS, GP, JC, and CD); statistical expertise (JA and JC); and literature search (JA and TS).

\section{Ethics}

The study was found to be exempted from review by the Human Studies Committee under Paragraph no. 4 of the Code of Federal Regulations 45 CFR 46.101(b). An IRB approval was received to collect data from medical records of patient. The project does not fall under HIPAA requirements because no protected health information is recorded or linked by code to data. The manuscript, its design, and implementation are in full adherence to the Declaration of Helsinki and all federal or state laws in the USA.

\section{References}

1 Ament JD, Stryjewski TP, Ciolino JB, Todani A, Chodosh J, Dohlman CH. Cost-Effectiveness of the Boston Keratoprosthesis. Am J Ophthalmol 2010; 149(2): 221-228.

2 Foster CS. Immunologic disorders of the conjunctiva, cornea, and sclera. In: Albert DM, Jakobiec FA (eds). Principles and practice of ophthalmology, 2nd ed Philadelphia, Pennsylvania: WB Saunders, 1999; 821-823.

3 Tugal-Tutkun I, Akova YA, Foster CS. Penetrating keratoplasty in cicatrizing conjunctival diseases. Ophthalmology 1995; 102: 576-585.

4 Solomon A, Ellies P, Anderson DF, Touhami A, Grueterich M, Espana EM et al. Long-term outcome of keratolimbal allograft with or without penetrating keratoplasty for total limbal stem cell deficiency. Ophthalmology 2002; 109: 1159-1166.

5 Dohlman $\mathrm{CH}$, Doane M. Keratoprosthesis in end-stage dry eye. Adv Exp Med Biol 1994; 350: 561-564.

6 Dohlman $\mathrm{CH}$, Terada H. Keratoprosthesis in pemphigoid and Stevens-Johnson syndrome. Adv Exp Med Biol 1998; 438: 1021-1025.

7 Yaghouti F, Nouri M, Abad JC, Power WJ, Doane MG, Dohlman $\mathrm{CH}$. Keratoprosthesis: preoperative prognostic categories. Cornea 2001; 20: 19-23.

8 Sayegh RR, Ang LPK, Foster S, Dohlman CH. The Boston Keratoprosthesis in Stevens-Johnson Syndrome. Am J Ophthalmol 2008; 145(3): 438-444.

9 Geerling G, Liu CSC, Collin JRO, Dart JKG. Costs and gains of complex procedures to rehabilitate end stage ocular surface disease. $\mathrm{Br} J$ Ophthalmol 2002; 86: 1220-1221.

10 Falcinelli G, Falsini B, Taloni M, Colliardo P, Falcinelli G. Modified osteo-odonto-keratoprosthesis for treatment of corneal blindness: long-term anatomical and functional outcomes in 181 cases. Arch Ophthalmol 2005; 123(10): 1319-1329.

11 Liu C, Okera S, Tandon R, Herold J, Hull C, Thorp S. Visual rehabilitation in end-stage inflammatory ocular surface disease with the osteo-odonto-keratoprosthesis: results from the UK. Br J Ophthalmol 2008; 92(9): 1211-1217.

12 Dohlman JG, Foster CS, Dohlman CH. Boston Keratoprosthesis in Steven-Johnson Syndrome: a case of using infliximab to prevent tissue necrosis. DJO 2009; 15(1).

13 Aldave AJ, Kamal KM, Vo RC, Yu F. The Boston type I keratoprosthesis: improving outcomes and expanding indications. Ophthalmology 2009; 116(4): 640-651.

14 Brown MM, Brown GC. How to interpret a healthcare economic analysis. Curr Opin Ophthalmol 2005; 16(3): 191-194.

15 Brown GC, Brown MM, Sharma S. Health care economic analyses. Retina 2004; 24(1): 139-146.

16 Dohlman $\mathrm{CH}$, Harissi-Dagher M, Khan BF, Sippel K, Aquavella JV, Graney JM. Introduction to the use of the Boston keratoprosthesis. Expert Rev Ophthalmol 2006; 1(1): 41-48.

17 Gold MR, Siegel JE, Russell LB, Weinstein MC. Cost-effectiveness in Health and Medicine. Oxford University Press: New York, 1996; 1-413.

18 Brown MM, Brown GC, Sharma S, Garrett S. Evidencebased medicine, utilities, and quality of life. Curr Opin Ophthalmol 1999; 10(3): 221-226. 
19 Hirneiss C, Neubauer AS, Niedermeir A, Messmer EM, Ulbig M, Kampik A. Cost utility for penetrating keratoplasty in patients with poor binocular vision. Ophthalmology 2006; 113: 2176-2180.

20 McCabe C, Claxton K, Culyer AJ. The NICE cost-effectiveness threshold-What it is and what that means. Pharmacoeconomics 2008; 26(9): 733-744.

21 Devlin N, Parkin D. Does NICE have a cost-effectiveness threshold and what other factors influence its decisions? A binary choice analysis. Health Econ 2004; 13(5): 437-452.

22 Summary of VELCADE Response Scheme. National Institute for Health and Clinical Excellence, October 2008. Available at http://www.nice.org.uk/nicemedia/pdf/ MyelomaDofHSummaryResponderScheme.pdf. Accessed 30 January 2010.

23 CPI Inflation Calculator. U.S. Bureau of Labor Statistics: Washington, DC, 2010 Available at http://www.bls.gov/ data/inflation_calculator.htm. Accessed on: March 3.

24 Busbee BG, Brown MM, Brown GC, Sharma S. Incremental cost-effectiveness of initial cataract surgery. Ophthalmology 2002; 109(3): 606-612.

25 Busbee BG, Brown MM, Brown GC, Sharma S. Cost-utility analysis of cataract surgery in the second eye. Ophthalmology 2003; 110(12): 2310-2317.

26 Sharma S, Brown GC, Brown MM, Hollands H, Shah GK. The cost-effectiveness of photodynamic therapy for fellow eyes with subfoveal choroidal neovascularization secondary to age-related macular degeneration. Ophthalmology 2001; 108(11): 2051-2059.

27 Losina E, Walensky RP, Kessler CL, Emrani PS, Reichmann WM, Wright EA et al. Cost-effectiveness of total knee arthroplasty in the United States. Arch Intern Med 2009; 169(12): 1113-1121.

28 Cummins JS, Tomek IM, Kantor SR, Furnes O, Engesaeter LB, Finlayson SR. Cost-effectiveness of antibiotic-impregnated bone cement used in primary total hip arthroplasty. J Bone Joint Surg Am 2009; 91(3): 634-641.
29 Griffin SC, Barber JA, Manca A, Sculpher MJ, Thompson SG, Buxton MJ et al. Cost effectiveness of clinically appropriate decisions on alternative treatments for angina pectoris: prospective observational study. BMJ 2007; 334(7594): 624-628.

30 Pinkerton SD, Holtgrave DR, Pinkerton HJ. Costeffectiveness of chemoprophylaxis after occupational exposure to HIV. Arch Intern Med 1997; 157(17): 1972-1980.

31 Dayton JD, Kanter KR, Vincent RN, Mahle WT. Costeffectiveness of pediatric heart transplantation. J Heart Lung Transplant 2006; 25(4): 409-415.

32 Kontodimopoulos N, Niakas D. An estimate of lifelong costs and QALYs in renal replacement therapy based on patients' life expectancy. Health Policy 2008; 86(1): 85-96.

33 Xie F, Lo NN, Tarride JE, O’Reilly D, Goeree R, Lee HP. Total or partial knee replacement? Cost-utility analysis in patients with knee osteoarthritis based on a 2-year observational study. Eur J Health Econ 2010; 11(1): 27-34.

34 Chang RW, Pellisier JM, Hazen GB. A cost-effectiveness analysis of total hip arthroplasty for osteoarthritis of the hip. JAMA 1996; 275(11): 858-865.

35 Mushlin AI, Mooney C, Holloway RG, Detsky AS, Mattson DH, Phelps CE. The cost-effectiveness of magnetic resonance imaging for patients with equivocal neurological symptoms. Int J Technol Assess Health Care 1997; 13(1): 21-34.

36 Faucett SC, Genuario JW, Tosteson AN, Koval KJ. Is prophylactic fixation a cost-effective method to prevent a future contralateral fragility hip fracture? J Orthop Trauma 2010; 24(2): 65-74.

37 Jacobson JJ, Schweitzer SO, Kowalski CJ. Chemoprophylaxis of prosthetic joint patients during dental treatment - a decision-utility analysis. Oral Surgery Oral Med Oral Pathol 1991; 72(2): 167-177.

38 Geerling G, Liu CS, Collin JR, Dart JK. Costs and gains of complex procedures to rehabilitate end stage ocular surface disease. Br J Ophthalmol 2002; 86(11): 1220-1221. 